\title{
Test de Actitud Prejuiciosa (TAP): Estudios de fiabilidad y evidencias de validez basadas en la estructura interna en autóctonos e inmigrantes
}

\author{
Antonio J. Rojas-Tejada ${ }^{1 *}$, Marisol Navas-Luque ${ }^{1}$, Pedro J. Pérez-Moreno ${ }^{2}$, \\ $\mathrm{M}^{\mathrm{a}}$ Isabel Cuadrado-Guirado ${ }^{1}$ y Oscar M. Lozano-Rojas ${ }^{2}$ \\ ${ }^{1}$ Universidad de Almería (España) \\ ${ }^{2}$ Universidad de Huelva (España)
}

\begin{abstract}
Resumen: Este trabajo se enmarca en el estudio del prejuicio en las poblaciones autóctona e inmigrante. El objetivo es llevar a cabo un estudio de las propiedades psicométricas de una nueva medida de la actitud de prejuicio para los diferentes grupos (mayorías y minorías), el Test de Actitud Prejuiciosa (TAP). El TAP se basa en una concepción tricomponente de la actitud prejuiciosa, considerando cogniciones, afectos e intenciones de conducta. Este trabajo muestra los resultados de las estimaciones de fiabilidad y el estudio de diversas fuentes de evidencias de validez: su estructura interna y relaciones con otras variables. La muestra estuvo formada por 1.967 participantes: 992 españoles, 500 rumanos y 475 ecuatorianos. La muestra fue seleccionada en 12 municipios con altas tasas de personas inmigrantes censadas, elegidas en cuatro comunidades autónomas de la zona mediterránea de España. El TAP presenta propiedades psicométricas adecuadas para su uso tanto en los grupos mayoritarios como minoritarios: una fiabilidad adecuada, una estructura interna sustantiva y relaciones con otras variables que son coherentes con el constructo en que se basa la prueba.

Palabras Clave: Prejuicio; grupos mayoritarios; grupos minoritarios; inmigrantes; fiabilidad; evidencias de validez.
\end{abstract}

\section{Introducción}

La mayoría de los modelos psicosociales del prejuicio (antiguos y contemporáneos) enfatizan su naturaleza emocional o evaluativa hacia los miembros de otros grupos. Otros lo conciben como una actitud negativa hacia un grupo social o sus miembros (Allport, 1954; Ashmore, 1970). Por otra parte, desde el modelo de actitud de los tres componentes (p.e. Breckler, 1984; Eagly y Chaiken, 1993; Harding, Kutner, Proshansky y Chein, 1969), se asume que el prejuicio racial o étnico es una actitud hacia los miembros de algún exogrupo étnico, cuyas respuestas evaluativas, predominantemente negativas, pueden ser divididas en tres clases: cognitivas, afectivas y conductuales. El prejuicio puede entenderse, por lo tanto, como un fenómeno multifacético que, además de tener un componente afectivo, tendrá componentes cognitivos y conductuales, que estarán interrelacionados de manera consistente (Devine, 1995).

Este planteamiento ha sido aplicado a los grupos mayoritarios o dominantes de cualquier sociedad. Sin embargo, los grupos étnicos minoritarios, especialmente los inmigrantes, apenas han sido estudiados en el área de las actitudes y el prejuicio. Existen numerosas teorías que describen el contenido y tratan de explicar el prejuicio de los grupos mayoritarios hacia los minoritarios, pero el punto de vista de éstos últimos ha sido mucho menos tratado, $y$, sobre todo, se ha tenido en cuenta en lo que se refiere a su percepción de este prejuicio, las repercusiones de tales actitudes en su autoesti-

* Dirección para correspondencia [Correspondence address]: Antonio J. Rojas Tejada. Facultad de psicología. Universidad de Almería, España. 04120 Almería (España).E-mail: arojas@ual.es
Title: Prejudice Attitude Test (PAT): Reliability and validity evidences based on the internal structure studies in autochthonous and immigrant Abstract: The framework of this research is the study of prejudice between autochthonous and immigrant people. Its aim is to conduct a study of the psychometric properties of a new measure of prejudice attitude for different groups (majority and minority groups), the Prejudice Attitude Test (PAT). The PAT is based on a conceptual design of attitude with three dimensions: cognitive, emotional and conative. This work shows the results of reliability estimates and the study of validity evidences (internal structure). Sample was formed by 1967 participants: there were 992 Spanish, 500 Romanian and 475 Ecuadorian people. The sample was selected in 12 municipalities with high rates of immigrant people in their census, chosen in four autonomous communities of Spain's Mediterranean zone. The PAT shows psychometric properties suitable for its use both in majority and in minority groups: an adequate reliability, a substantive internal structure that are consistent with the construct underlying the test.

Key Words: Prejudice; majority groups; minority groups; immigrants; reliability; evidence of validity.

ma, salud mental, identidad y formas de enfrentarse a la discriminación (p.e., Quiles y Leyens, 2003; Swim y Stangor, 1998).

Si bien numerosos autores han reclamado la necesidad de conocer también las actitudes de los inmigrantes o de los grupos minoritarios hacia la población autóctona o dominante (p.e., Judd, Park, Ryan, Brauer, y Kraus, 1995; Monteith y Spicer, 2000; Shelton, 2000), apenas existen estudios psicosociales que lleven a la práctica este reclamo, y los que se han realizado han utilizado dos procedimientos principales: a) adaptar a los grupos minoritarios instrumentos ya existentes para medir el prejuicio de los grupos mayoritarios, simplemente invirtiendo el sentido de los ítems (especialmente afroamericanos en EEUU) (p.e., Brigham, 1993; Ellison y Powers, 1996; Schuman, Steeh, Bobo y Krysan, 1997); y b) empleando técnicas cualitativas como el análisis de contenido de los discursos o los relatos de los participantes (p.e., Johnson y Lecci, 2003; Monteith y Spicer, 2000), con el fin elaborar modelos sobre las actitudes intergrupales partiendo de las experiencias de las minorías. Por tanto, uno de los problemas fundamentales que se plantea cuando se pretende medir las actitudes intergrupales de mayorías y minorías en interacción es la necesidad de utilizar un instrumento de medida que permita captar las peculiaridades de la actitud en cada grupo, pero que permita hacer comparaciones intergrupales.

En la presente investigación se muestra el resultado de elaborar una medida de prejuicio, el TAP, que permita aprehender dicha actitud en el grupo minoritario de inmigrantes y en el mayoritario de autóctonos, partiendo de una posición integradora que concibe el prejuicio como una actitud social, cuya evaluación requiere recoger la percepción que se tiene 
del exogrupo, las emociones que suscita y la intención de comportarse ante él.

En el TAP la variable prejuicio se ha operacionalizado a partir de la combinación de tres variables: opinión sobre el exogrupo o estereotipo (componente cognitivo del prejuicio), emociones (componente afectivo) y distancia social preferida con el exogrupo (componente conativo o de intención conductual).

Para el componente cognitivo (opinión sobre el exogrupo) se diseñó una medida ligeramente diferente a la utilizada tradicionalmente: frente a la medida individual, en la que las personas describen a los miembros de un exogrupo en términos de rasgos o características individuales que éstos poseen por pertenecer al grupo, se ha utilizado una medida con características grupales o culturales para describir al exogrupo. Esta medida hace referencia a una serie de ámbitos socioculturales del exogrupo contemplados en el Modelo Ampliado de Aculturación Relativa -MAAR- (p.e., Navas y Rojas, 2010; Navas, Rojas, Pumares, Lozano y Cuadrado, 2010; Rojas, Navas, Lozano y Gómez, 2010; Rojas, Navas, Lozano y Pérez, 2011): político, de bienestar social, laboral (componente cognitivo periférico), económico, social (componente cognitivo intermedio), familiar, religioso y de valores (componente cognitivo central). El componente afectivo del prejuicio (emociones hacia el exogrupo) consta de emociones positivas (admiración, simpatía y respeto) y emociones negativas consideradas "sutiles" (desconfianza, incomodidad, inseguridad e indiferencia) por los investigadores del prejuicio moderno, sutil, latente o aversivo (p.e. Dovidio y Gaertner, 1986; McConahay, 1983; Pettigrew y Meertens, 1992 y 1995). Finalmente, el componente conativo/conductual de la actitud prejuiciosa se midió con la adaptación de una escala clásica de distancia social (p.e., Bogardus, 1927). Ante la imposibilidad de medir directamente la discriminación como conducta observable, consideramos que la intención de conducta manifestada por una persona hacia el mantenimiento o evitación de relaciones con miembros del exogrupo (la distancia social preferida) podría considerarse un indicador del componente conativo/conductual de la actitud prejuiciosa. Esta variable hace referencia al tipo de relación (más o menos cercano o distante) que los participantes prefieren mantener con miembros del exogrupo.

El objetivo de este trabajo es estimar la fiabilidad del TAP y mostrar evidencias de validez basadas en la estructura interna del mismo. Para ello y basándonos en los últimos Standards de la AERA/APA/NCME (1999) sobre evidencias de validez (basadas en la estructura interna), se contrastará un modelo de estructura latente multigrupo del instrumento.

\section{Método}

\section{Participantes}

Participaron 1967 personas, de las cuales 992 eran españolas autóctonas y 975 eran inmigrantes. De los españoles,
499 respondían a las preguntas del cuestionario teniendo como referencia al grupo de inmigrantes rumanos (Grupo AR), y 493 al grupo de inmigrantes ecuatorianos (Grupo $\mathrm{AE}$ ); de los inmigrantes, 500 son de origen rumano (Grupo IR) y 475 de origen ecuatoriano (Grupo IE). Todas ellas residían en 12 municipios (de cuatro comunidades autónomas del Mediterráneo) con altas tasas de recepción de inmigrantes de estos orígenes (más del $2 \%$ de personas inmigrantes de cada uno de estos orígenes sobre el total de la población censada). Concretamente, fueron municipios de la Comunidad Autónoma Andaluza en el 59\% de los casos, Murciana en el $13.5 \%$, Valenciana en el $13.9 \%$ y Catalana en el $13.5 \%$. Las muestras de autóctonos fueron seleccionadas mediante muestreo polietápico: 1) selección por expertos de municipios de alta recepción de inmigrantes (los municipios o agrupaciones de municipios adyacentes con mayor proporción de inmigrantes rumanos y ecuatorianos censados, teniendo como criterio de inclusión que al menos un 5\% del censo estuviese constituido por inmigrantes y al menos un $2 \%$ de alguna de ambas nacionalidades); 2) estratificación por sexo y edad (con afijación proporcional), y 3) rutas aleatorias para la recogida de la muestra en las distintas zonas diana. El error muestral asumido para el total de la muestra fue del $\pm 3.2 \%(\mathrm{p}=\mathrm{q}=.5$; nivel de confianza: $95.5 \%)$, y para las submuestras de autóctonos que evaluaban a rumanos (Grupo AR) y ecuatorianos (Grupo AE) en las mismas condiciones y parámetros estadísticos, el error muestral se eleva al $\pm 4.3 \%$ para cada una. Para las muestras de inmigrantes se optó por un muestreo por cuotas realizado en los mismos municipios que las muestras de autóctonos en el que, al menos, se seleccionaran a grupos equivalentes a los que se obtendrían con un muestreo aleatorio (aproximadamente un $50 \%$ de varones y otro tanto de mujeres en cada muestra).

La edad media de los autóctonos supera los 40 años: en el Grupo AR 45 años (con DT = 16.7), y en el Grupo AE 45.1 (DT =16.8). Las muestras de inmigrantes presentan una edad media para el Grupo IR de 34 años y de 32 para el Grupo IE (con una DT de 10.6 para ambos grupos). Todas las muestras estuvieron equilibradas en función del sexo (alrededor del 50\%). El trabajo de campo de esta investigación se realizó cuando los rumanos todavía eran considerados, en términos legales, inmigrantes extracomunitarios.

\section{Variables e Instrumentos}

\section{Prejuicio/Actitud Prejuiciosa.}

La medida del componente cognitivo (opinión sobre el exogrupo o estereotipo) de la actitud prejuiciosa estaba compuesta por 8 ítems que reflejan los ámbitos del MAAR (ver tabla 1): político, de bienestar social, laboral (componente cognitivo periférico), económico, social (componente cognitivo intermedio), familiar, religioso y de valores (componente cognitivo central). 
Tabla 1. Ítems del Test de Actitud Prejuiciosa (TAP).

Componente cognitivo (opinión sobre el exogrupo): Valore las siguientes cuestiones empleando la siguiente escala de respuesta: 1: Muy malos; 2: Malos; 3: Ni malos ni buenos; 4: Buenos; 5: Muy buenos.

Piense en las personas (del exogrupo) y dígame, ¿Cómo cree Vd. que son....

ITEM 1. Su sistema político y de gobierno (forma en que se eligen los gobiernos, forma en que funcionan, participación política, leyes, etc. ).

ITEM 2. Su sistema de bienestar social (educación, sanidad y servicios sociales).

ITEM 3. Sus formas de trabajar (ritmo de trabajo, horario, condiciones laborales -p.e., desempleo, eventualidad, etc.).

ITEM 4. Sus hábitos de consumo y economía doméstica (productos que compran, tipo de comida que consumen, economía familiar -p.e., dinero que gastan y ahorran, forma de administrar lo que tienen, etc.).

ITEM 5. Sus relaciones sociales (forma de relacionarse, lugares habituales de relación social, uso del tiempo libre y formas de divertirse, etc.).

ITEM 6. Sus relaciones familiares (forma de relacionarse con la pareja, hijos-as, las personas mayores de la familia, reparto de roles o funciones, etc.).

ITEM 7. Sus creencias y prácticas religiosas (creencias, practicas y cumplimiento personal de obligaciones o prohibiciones religiosas).

ITEM 8. Sus valores (respeto a las personas mayores, forma de educar a los hijos-as, igualdad entre hombres y mujeres, papel de la religión en la vida, etc.).

Componente afectivo (emociones): En general, ¿̇en qué grado ha sentido Vd. las siguientes emociones hacia las personas (del exogrupo)? Utilice la siguiente escala para responder: 1: Nada; 2: Poco; 3: Algo; 4: Bastante; 5: Mucho.

ITEM 9. Admiración; ITEM 10. Desconfianza; ITEM 11. Simpatía; ITEM 12. Incomodidad; ITEM 13. Inseguridad; ITEM 14. Respeto; ITEM 15. Indiferencia.

Componente conativo/conductual (distancia social):

ITEM 16. Si pudiera elegir, ¿hasta dónde estaría usted dispuesto/a a llegar en su relación con personas (del exogrupo)? (se pueden señalar varias):

a. A formar una familia con una persona (del exogrupo) o tener una pareja (del exogrupo).

b. A que mis hijos o hijas eligieran como pareja a una persona (del exogrupo).

c. A tener amigos-as (del exogrupo).

d. A tener vecinos-as o compañeros-as de trabajo (del exogrupo).

e. Prefiero no tener relación con personas (del exogrupo).

Los participantes tenían que indicar su opinión sobre cada uno de los ámbitos del exogrupo mencionados, utilizando una escala de respuesta que oscilaba desde 1 (muy malo) hasta 5 (muy bueno). Las puntuaciones en este componente se invierten para que las puntuaciones altas en esta medida indiquen un mayor prejuicio hacia el exogrupo ( $\mathrm{y}$ a la inver$\mathrm{sa}$, puntuaciones bajas indiquen menos prejuicio).

Las emociones hacia el exogrupo (componente afectivo del prejuicio) se midieron mediante 7 ítems (ver anexo): 3 ítems de emociones positivas (admiración, simpatía y respeto) y 4 ítems de emociones negativas sutiles (desconfianza, incomodidad, inseguridad e indiferencia). Este listado de emociones, con alguna ligera variación, se había utilizado en trabajos anteriores (p.e., Navas, García, Rojas, Pumares y Cuadrado, 2006). Los participantes debían indicar "el grado en el que habían sentido cada una de esas emociones hacia las personas del exogrupo", utilizando para ello una escala de frecuencia que oscilaba desde 1 (nada) hasta 5 (mucho). Las puntuaciones en emociones positivas se han invertido para que las puntuaciones altas indiquen un mayor grado de emociones negativas hacia el exogrupo y, por tanto, mayor prejuicio exogrupal y viceversa.

El componente conativo o de intención conductual (distancia social) se midió con una adaptación de una escala clásica de distancia social (p.e., Bogardus, 1927): un solo ítem (ver anexo) en el que se pide a los participantes que indiquen "hasta dónde estarían dispuestos a llegar en su relación con miembros del exogrupo". Sus cinco alternativas de respuesta oscilan desde la preferencia por mantener una relación muy estrecha con personas del exogrupo ("A formar una familia con una persona del exogrupo", valorada con 1 punto), has- ta la preferencia por "no tener relación con personas del exogrupo" (valorada con 5 puntos), pasando por opciones intermedias. Los participantes podían señalar varias opciones, empleándose para los análisis la opción más inclusiva (la que indicaba una preferencia por mantener una relación más íntima con personas del exogrupo).

La medida de prejuicio del TAP se obtiene sumando cada dimensión ponderada por el número de ítems de cada componente.

\section{Procedimiento}

El TAP se incluyó en un cuestionario más amplio donde se preguntaba por distintas variables en el marco del estudio del proceso de aculturación de inmigrantes y autóctonos. Este cuestionario se administró de forma individualizada por personal formado al efecto. Para los grupos AR y AE se realizó la aplicación en el propio domicilio de la persona seleccionada; para el resto de grupos bien en el domicilio particular de la persona, bien en asociaciones sindicales o de inmigrantes, o en alguna ONG. Para todos los grupos el idioma utilizado en el cuestionario fue el español, si bien los encuestadores de los grupos IR e IE eran del mismo origen nacional que los participantes inmigrantes.

\section{Análisis estadístico}

El análisis de datos se ha realizado sobre la matriz de casos completos, es decir, aquellos que no cuentan con valores perdidos. Se consideró valor perdido a las respuestas "No sabe/No contesta" al considerar que no cuentan con un valor sustantivo en el contenido medido en cada ítem. Se ha 
llevado a cabo un análisis descriptivo de la puntuación TAP, así como la estimación de su fiabilidad (índices de discriminación de los ítems y coeficiente alfa de Cronbach) en cada submuestra, empleando el programa SPSS 15.0 (SPSS Inc., 2006). La estructura teórica del TAP se ha puesto a prueba mediante Análisis Factorial Confirmatorio Multigrupo. Primero se comprobó el ajuste del modelo en cada una de las muestras, probando posteriormente la invarianza métrica (Elosua, 2005): el grado en que el modelo ajusta en los distintos grupos, igualando entre ellos los parámetros de la relación entre cada ítem y cada factor (Byrne, 2006). Se empleó el método de estimación de Máxima Verosimilitud y la prueba $\chi^{2}$ Robusta de Satorra-Bentler ( $\mathrm{S}-\mathrm{B} \chi^{2}$, Satorra y Bentler, 1994); debido a la sensibilidad de ésta al tamaño de la muestra y las desviaciones con respecto a los supuestos de linealidad, normalidad multivariada y aditividad (Jöreskog y Sörbom, 1989) se empleará la razón $\mathrm{S}-\mathrm{B} \chi^{2} / \mathrm{gl}$ (el ajuste es adecuado si resulta inferior a 3.0; Carmines y McIver, 1981). Se interpretará el índice de ajuste global GFI (Goodness-of-FitIndex, índice de bondad de ajuste, Jöreskog y Sörbom, 1989), y los índices comparativos NFI (Normed-Fit-Index, índice de ajuste normativo), NNFI (Non-Normed-Fit-Index, índice de ajuste no-normativo), CFI (Comparative-Fit-Index, índice de ajuste comparativo; Bentler, 1990) e IFI (Incremental-Fit-Index, índice de ajuste incremental; Bollen, 1989). Valores iguales o superiores a .90 de dichos índices se interpretan como propios de un ajuste adecuado. También se calculó el valor RMSEA (Root Mean Square Error of Approximation, raíz cuadrática media del error de aproximación; Byrne, 2001; Steiger, 1990), indicando un ajuste adecuado todo valor igual o inferior a 0,08 (Browne y Cudeck, 1993). Para este análisis se empleó el programa EQS 6.0 (Bentler, 2005).

\section{Resultados}

\section{Análisis de ítems y fiabilidad}

Los índices de discriminación de los ítems (mayores de .20 en todos los grupos, excepto el ítem 1 en las muestras AE e IR y el ítem 3 en la muestra IE) y coeficientes de fiabilidad estimados (alfa de Cronbach, con valores comprendidos entre .74 y .89) de los diversos componentes del TAP para todos los grupos se muestran en la tabla 2, ofreciendo unos resultados más que aceptables.

Tabla 2. Estadísticos descriptivos de la TAP, índices de discriminación de los ítems, correlaciones y coeficientes alfa de los componentes para cada muestra.

\begin{tabular}{|c|c|c|c|c|c|c|c|c|}
\hline TAP & AR & & $\mathrm{AE}$ & & IR & \multicolumn{3}{|c|}{ IE } \\
\hline $\mathrm{N}$ & 225 & & 243 & & 352 & \multicolumn{3}{|c|}{363} \\
\hline Elementos & 16 & & 16 & & 16 & \multicolumn{3}{|c|}{16} \\
\hline Media & 8.83 & & 8.42 & & 6.90 & \multicolumn{3}{|c|}{7.16} \\
\hline Desv. Típica & 2.55 & & 1.85 & & 1.57 & \multicolumn{3}{|c|}{1.53} \\
\hline Minimo & 4.61 & & 4.43 & & 3.29 & \multicolumn{3}{|c|}{3.70} \\
\hline Maximo & 15.00 & & 13.84 & & 11.09 & \multicolumn{3}{|c|}{11.98} \\
\hline Alpha Cronbach & .89 & & .80 & & .78 & \multicolumn{3}{|c|}{.71} \\
\hline Componente Cognitivo (CC) & AR & & $\mathbf{A E}$ & & IR & \multicolumn{3}{|c|}{ IE } \\
\hline ITEM 1 (periférico) & .307 & & .180 & & .186 & \multicolumn{3}{|c|}{.225} \\
\hline ITEM 2 (periférico) & .332 & & .201 & & .418 & \multicolumn{3}{|c|}{.245} \\
\hline ITEM 3 (periférico) & .380 & & .219 & & .335 & \multicolumn{3}{|c|}{.187} \\
\hline ITEM 4 (intermedio) & .513 & & .246 & & .449 & \multicolumn{3}{|c|}{.345} \\
\hline ITEM 5 (intermedio) & .663 & & .486 & & .465 & \multicolumn{3}{|c|}{.409} \\
\hline ITEM 6 (central) & .583 & & .489 & & .489 & \multicolumn{3}{|c|}{.404} \\
\hline ITEM 7 (central) & .565 & & .452 & & .429 & \multicolumn{3}{|c|}{.333} \\
\hline ITEM 8 (central) & .632 & & .479 & & .474 & \multicolumn{3}{|c|}{.425} \\
\hline Alpha Cronbach & .83 & & .75 & & .77 & \multicolumn{3}{|c|}{.68} \\
\hline Componente Afectivo (CA) & AR & & $\mathbf{A E}$ & & IR & \multicolumn{3}{|c|}{ IE } \\
\hline ITEM 1 (positiva) & .463 & & .423 & & .423 & \multicolumn{3}{|c|}{.423} \\
\hline ITEM 2 (positiva) & .627 & & .592 & & .592 & \multicolumn{3}{|c|}{.592} \\
\hline ITEM 3 (positiva) & .447 & & .586 & & .586 & \multicolumn{3}{|c|}{.586} \\
\hline ITEM 4 (negativa sutil) & .621 & & .374 & & .374 & \multicolumn{3}{|c|}{.374} \\
\hline ITEM 5 (negativa sutil) & .705 & & .487 & & .487 & \multicolumn{3}{|c|}{.487} \\
\hline ITEM 6 (negativa sutil) & .626 & & .377 & & .377 & \multicolumn{3}{|c|}{.377} \\
\hline ITEM 7 (negativa sutil) & .597 & & .366 & & .366 & \multicolumn{3}{|c|}{.366} \\
\hline Alpha Cronbach & .85 & & .78 & & .64 & \multicolumn{3}{|c|}{.63} \\
\hline Componente Conativo (Cco) & AR & & $\mathbf{A E}$ & & IR & \multicolumn{3}{|c|}{ IE } \\
\hline ITEM 1 & .583 & & .422 & & .342 & & & \\
\hline Correlaciones & $\mathbf{A R}$ & & $\mathbf{A E}$ & & IR & & & \\
\hline & $\mathrm{CC}$ & CA & $\mathrm{CC}$ & CA & $\mathrm{CC}$ & CA & $\mathrm{CC}$ & CA \\
\hline Componente Afectivo (CA) & $.55^{* *}$ & & $.29 *$ & & $.36^{* *}$ & & $.26 * *$ & \\
\hline Componente Conativo (CCo) & $.43^{* *}$ & $.58 * *$ & $.17 * *$ & $.48^{* *}$ & $.22 * *$ & $.35^{* *}$ & $.30 * *$ & $.13^{*}$ \\
\hline
\end{tabular}


Asimismo, los tres componentes del TAP (cognitivo, afectivo y conativo) aparecen correlacionados de forma estadísticamente significativa y en la dirección esperada en todas las muestras del estudio (ver tabla 2).

\section{Descriptivos del TAP}

La medida obtenida en el TAP oscila entre un valor mínimo de 3 y un valor máximo de 15 . El valor medio de prejuicio es mayor en el caso de la muestra de personas autóctonas, siendo de 8.83 (DT=2.55) en el Grupo AR y 8.42 (DT=1.85) en el Grupo AE. Para las muestras de inmigrantes, estos valores son menores (en el Grupo IR de $6.90 \mathrm{y}$ DT=1.57, y en el Grupo IE de 7.66, con DT=2.00). Entre los cuatro grupos existen diferencias estadísticamente significativas $\left(\mathrm{F}^{*}\right.$ de Brown-Forsythe $(3 ; 755,512)=65.24$; $\mathrm{p}<.0001)$. Se contrastó un grupo ortogonal de comparaciones planificadas que comparaba las muestras de autóctonos entre sí, las muestras de inmigrantes entre sí, y el promedio de las muestras de autóctonos contra el de las muestras de inmigrantes. Para mantener $\alpha=.05$ en la familia de comparaciones se empleó un valor alfa por comparación de .017 $\left(\alpha_{\mathrm{PC}}=.017\right)$ resultante de aplicar la corrección de Bonferroni. Los resultados indican que no existe evidencia de diferencias estadísticamente significativas entre las muestras de autóctonos $(\mathrm{t}(406,1)=1.953 ; \mathrm{p}=.051$-bilateral-, $d$ de Cohen=.19), ni entre las muestras de inmigrantes $(\mathrm{t}(710,764)=2.216 ; \mathrm{p}=.027$ -bilateral-, $d$ de Cohen=.17), pero sí entre el promedio de los autóctonos y el de los inmigrantes $(\mathrm{t}(663,006)=13.410$; $\mathrm{p}<.001$-bilateral-, $d$ de Cohen=.86). Se contrastó un segundo grupo ortogonal (con idéntico procedimiento de corrección del Error Tipo I) sobre las posibles diferencias entre las muestras AR e IR, entre las muestras AE e IE, y entre el promedio de AR e IR contra el promedio de AE e IE. En este caso la tercera comparación ofrece resultados estadísticamente no significativos $(\mathrm{t}(663,006)=.623 ; \mathrm{p}=.534$ - bilateral-, $d$ de Cohen=.01), siendo estadísticamente significativas tanto las diferencias entre AR e IR $(t(333,150)=10.152$; $\mathrm{p}<.001$-bilateral-, $d$ de Cohen=.96) como las diferencias entre AE e IE $(\mathrm{t}(541,343)=10.152 ; \mathrm{p}=.001$ - bilateral-, $d$ de Cohen $=.76$ ). Por lo tanto no contamos con evidencia de diferencias en el prejuicio entre ambas muestras de autóctonos ni entre ambas muestras de inmigrantes, pero sí es mayor el prejuicio en los autóctonos que en los inmigrantes.

\section{Estudio de evidencias de validez}

El Análisis de la estructura interna del test se comprobó mediante análisis factorial confirmatorio el ajuste del modelo factorial de segundo orden representado en la Figura 1 (no se muestran las representaciones de errores de medida para mayor simplicidad).

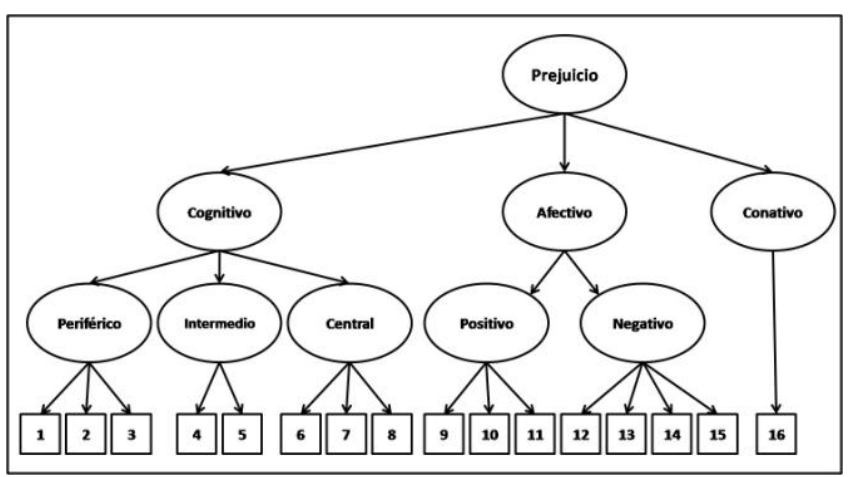

Figura 1. Estructura del TAP.

Primero se estimó el ajuste de dicho modelo en cada muestra. En la tabla 3 se muestran los índices de ajuste obtenidos. En el Grupo AR todos los índices excepto la prueba de $\mathrm{S}-\mathrm{B} \chi^{2}$ son adecuados $\left(\mathrm{S}-\mathrm{B} \chi^{2}(97)=140,89 ; \mathrm{p}<.05\right.$; siendo $\mathrm{S}$ $\mathrm{B} \chi^{2} / \mathrm{gl}<3.0$, y GFI, NFI, NNFI, CFI e IFI mayores que 0,90; RMSEA<.08). En el Grupo AE, el test $\mathrm{S}-\mathrm{B} \chi^{2}$ es adecuado $\left(\mathrm{S}-\mathrm{B} \chi^{2}(97)=119.02 ; \mathrm{p}>.05\right)$ como el resto de índices, excepto el NFI $<.90$. En el Grupo IR el test de $\mathrm{S}-\mathrm{B} \chi^{2}$ resulta estadísticamente significativo $\left(\mathrm{S}-\mathrm{B} \chi^{2}(97)=217,23 ; \mathrm{p}<.05\right)$ al igual que los índices GFI y NFI, siendo el resto de índices adecuados. En el Grupo IE se obtiene un valor S-B $\chi^{2}$ inadecuado $\left(\mathrm{S}-\mathrm{B} \chi^{2}(97)=180.67 ; \mathrm{p}<.05\right)$ al igual que losíndices NFI y NNFI (ambos inferiores a .90), resultando el resto de índices indicadores de un ajuste adecuado. Teniendo en cuenta que los índices que muestran un ajuste poco adecuado son los sensibles a las desviaciones de la normalidad multivariada y al tamaño de las muestras, nada se opone a interpretar que el modelo propuesto presenta un ajuste adecuado en las cuatro muestras.

Tabla 3. Índices de ajuste del modelo en cada una de las muestras.

\begin{tabular}{|c|c|c|c|c|c|c|c|c|c|c|c|}
\hline & $\mathrm{S}-\mathrm{B} \chi^{2}$ & gl & $p$ & $\mathrm{~S}-\mathrm{B} \chi^{2} / \mathrm{gl}$ & GFI & NFI & NNFI & CFI & IFI & RMSEA & $90 \%$ RMSEA \\
\hline $\mathrm{AR}$ & 140.89 & 97 & .002 & 1.45 & 0.912 & 0.900 & 0.957 & 0.966 & 0.966 & 0.045 & $0.027-0.060$ \\
\hline $\mathrm{AE}$ & 119.02 & 97 & .064 & 1.23 & 0.938 & 0.888 & 0.971 & 0.977 & 0.977 & 0.031 & $0.000-0.048$ \\
\hline IR & 217.23 & 97 & .000 & 2.24 & 0.898 & 0.871 & 0.904 & 0.923 & 0.924 & 0.059 & $0.049-0.070$ \\
\hline IE & 180.67 & 97 & .000 & 1.86 & 0.929 & 0.832 & 0.891 & 0.912 & 0.914 & 0.049 & $0.038-0.060$ \\
\hline Invarianza & 850.75 & 433 & .000 & 1.96 & 0.900 & 0.838 & 0.902 & 0.912 & 0.913 & 0.029 & $0.026-0.031$ \\
\hline
\end{tabular}

Finalmente, se comprobó la invarianza métrica mediante el modelo multigrupo con saturaciones factoriales equiparadas entre las muestras. Puede comprobarse en la Tabla 3 que, a excepción del valor NFI, el modelo multigrupo resultó adecuado. 


\section{Discusión y conclusiones}

El objetivo fundamental de este trabajo era presentar las propiedades métricas de un test desarrollado para la medida del prejuicio o la actitud prejuiciosa (TAP) en grupos mayoritarios (autóctonos) y minoritarios (inmigrantes rumanos y ecuatorianos). El TAP ha sido elaborado partiendo del modelo de los tres componentes de la actitud (p.e., Breckler, 1984; Eagly y Chaiken, 1993; Harding, Proshansky, Kutner y Chein, 1969). Nuestro trabajo pretende solucionar uno de los problemas fundamentales que se plantea en la investigación psicosocial cuando se pretenden medir las actitudes intergrupales de mayorías y minorías en interacción: la necesidad de utilizar un instrumento de medida que permita captar las peculiaridades de la actitud prejuiciosa en cada grupo, pero también suficientemente similar para poder hacer comparaciones intergrupales.

Las medidas que proporciona el TAP han resultado sensibles a los grupos a los que se ha aplicado tanto si éstos eran considerados mayoritarios o minoritarios. Así, hemos obtenido puntuaciones moderadas o bajas de prejuicio en los cuatro grupos del estudio, pero los grupos mayoritarios (AR y AR) presentan puntuaciones significativamente más elevadas que los grupos minoritarios (IR e IE), como cabría esperar teóricamente. Nuestros resultados revelan, por tanto, que los autóctonos muestran mayores niveles de prejuicio exogrupal que los inmigrantes, si bien no existen diferencias estadísticamente significativas entre los dos grupos de autóctonos (AR y AE), ni entre los dos grupos de inmigrantes (IR e IE) en sus niveles de prejuicio.

Respecto a la estimación de la fiabilidad del TAP ha mostrado un funcionamiento adecuado en función de los valores obtenidos en todos los grupos del estudio (autóctonos e inmigrantes), ya que alcanzan el nivel óptimo señalado para el desarrollo de este tipo de instrumentos de medida. Además hay que señalar la existencia de correlaciones estadísticamente significativas entre los tres componentes del TAP, tanto en autóctonos como en inmigrantes, y siempre en la dirección esperada teóricamente. Es decir, que los tres componentes de la actitud prejuiciosa (cognitivo, afectivo y de intención de conducta) aparecen positivamente relacionados, de manera que a peor opinión del exogrupo, más emociones negativas se experimentan hacia él y mayor distancia social se prefiere mantener con él. También se encuentran diferencias estadísticamente significativas al comparar entre sí a autóctonos que evalúan a rumanos frente a rumanos que evalúan a autóctonos, al igual que ocurre entre

\section{Referencias}

Allport, G. W. (1954). The nature of prejudice. Reading, MA: Addison-Wesley. APA/AERA/NCME (American Psychological Association, American Educational Research Association y National Council on Measurement in Education) (1999). Standards for educational and psychological testing. Washinton, DC: American Psychological Association.

Amir, Y. (1969). Contact hypothesis in ethnic relations. Psychological Bulletin, 71, 319-342. autóctonos que evalúan a ecuatorianos frente a ecuatorianos que evalúan a autóctonos.

Asimismo, la estructura interna del TAP se ajusta a una medida única de la actitud prejuiciosa que cuenta con tres componentes que pueden ser interpretados de manera independiente. Esta estructura, por otra parte, se ha mostrado adecuada en las cuatro muestras. Igualmente puede afirmarse que el peso de cada ítem en las respectivas subescalas, así como de cada subescala en la medida global, es equivalente en las cuatro muestras.

Por tanto, a la luz de los resultados sobre las medidas que proporciona el TAP, la estimación de los coeficientes de fiabilidad obtenidos para las distintas muestras y los resultados procedentes de las evidencias de validez, podemos avalar que el TAP posee unas propiedades psicométricas adecuadas para su uso con grupos mayoritarios (autóctonos) y minoritarios (inmigrantes), es decir, para medir prejuicio hacia el exogrupo, independientemente de que éste sea un grupo mayoritario o minoritario. En este sentido, es importante señalar que la utilización de una única medida de prejuicio que sea aplicable (con garantías psicométricas y con apoyo teórico) a población autóctona e inmigrante no es un hecho frecuente en la investigación psicosocial, por lo que los resultados encontrados en nuestro trabajo nos parecen doblemente interesantes.

Quedan para futuras investigaciones realizar otros estudios de evidencias de validez del TAP, como, por ejemplo, su relación con otros constructos. Una limitación del estudio es que las muestras de autóctonos e inmigrantes no han sido seleccionadas siguiendo la misma técnica (muestreo probabilístico polietápico por conglomerados y rutas aleatorias, en el caso de los autóctonos y muestreo por conglomerados y cuotas en el caso de los inmigrantes). Se optó por el muestreo no probabilístico de inmigrantes debido a la subrepresentación de éstos en el censo (Pumares, García y Asensio, 2006). Así queda para futuros trabajos perfeccionar el procedimiento de muestreo de inmigrantes.

Agradecimientos.- Este trabajo ha sido realizado gracias a varios proyectos, uno financiado por la Secretaría de Estado de Universidades, Investigación y Desarrollo del Plan Nacional I+D+I (Referencia SEJ2004-07369/PSIC), otro financiado por la Consejería de Gobernación (Dirección General de Políticas Migratorias) de la Junta de Andalucía (Referencia 2005/270) y un proyecto de Excelencia financiado por la Consejería de Innovación, Ciencia y Empresa de la Junta de Andalucía (Referencia P09-SEJ-4657).

Ashmore, R. D. (1970). The problem of the intergroup prejudice. En B. Collins, Social Psychology. Reading, MA: Addison-Wesley.

Bentler, P. M. (1990). Comparative fit indexes in structural models. Psychological Bulletin, 107, 238-246.

Bentler, P. M. (2005). EQS 6 Structural equations program manual. Encino, CA: Multivariate Software. 
Bogardus, E. S. (1927). Race friendliness and social distance. Journal of Applied Sociology, 11, 272-287.

Bollen, K. A. (1989). A new incremental fit index for general structural equation models. Sociological Methods \& Research, 17, 303-316.

Breckler, S. J. (1984). Empirical validation of affect, behavior, and cognition as distinct components of attitude. Journal of Personality and Social Psychology, 47 (6), 1191-1205.

Brigham, J. C. (1993). College students' racial attitudes. Journal of Applied Social Psychology, 23, 1933-1967.

Brown, R. J. (1984). The role of similarity in intergroup relations. En H. Tajfel, The Social Dimension (Vol. 2). Cambridge: Cambridge University Press.

Browne, M. W., y Cudek, R. (1993). Alternative ways of assessing model fit. En K. A. Bollen, y J. S. Long, Testing structural equation models (pp. 136162). Newbury Park, CA: Sage.

Byrne, B. M. (2001). Structural equation modeling with AMOS: basic concepts, applications, and programming. Mahwah, NJ: Lawrence Erlbaum Associates.

Byrne, B. M. (2006). Structural Equation Modeling with EQS. Basic Concepts, Applications, and Programming (2 ed.). Mahwah, NJ: Lawrence Erlbaum Associates.

Carmines, E. G., y McIver, J. P. (1981). Analyzing models with unobserved variables. En G. W. Bohrnstedt, y E. F. Borgatta, Social measurement: Current issues (pp. 122-133). Beverly Hills, CA: Sage.

Devine, P. G. (1995). Prejudice and out-group perception. En A. Tesser, Advanced Social Psychology (pp. 467-524). New York: McGraw-Hill.

Dovidio, J. F., y Gaertner, S. I. (1986). Prejudice, discrimination, and racism. New York: Academic Press.

Eagly, A. H., y Chaiken, S. (1993). The Psychology of Attitudes. San Diego, CA: Harcourt Brace Jovanovich.

Ellison, C. G., y Powers, D. A. (1996). The contact hypothesis and racial attitudes among Blacks. Social Science Quarterly, 75, 385-400.

Elosua, P. (2005). Evaluación progresiva de la invarianza factorial entre las versiones original y adaptada de una escala de autoconcepto. Psicothema, 17 (2), 356-362.

Gómez-Berrocal, C., y Navas, M. (2000). Predictores del prejuicio manifiesto y sutil hacia los gitanos. Revista de Psicología Social, 15 (1), 3-30.

Harding, J., Proshansky, H., Kutner, B., y Chein, I. (1969). Prejudice and ethnic relations. En G. Lindzey, y E. Aronson, Handbook of Social Psychology (Vol. 5, pp. 1-76). Reading, MA: Addison-Wesley.

Hogg, M. A. (1992). The Social Psychology of Groups Cohesiveness: From Atraction to Social Identity. Nueva York: Harvester Wheatsheaf.

Johnson, J. D., y Lecci, L. (2003). Assessing anti-white attitudes and predicting perceived racism: The Johnson-Lecci Scale. Personality and Social Psychology Bulletin, 29 (3), 299-312.

Jöreskog, K. G., y Sörbom, D. (1989). LISREL 7 User's Reference Guide. Chicago, IL: Scientific Software, Inc.

Judd, C. M., Park, B., Ryan, C. S., Brauer, M., y Kraus, S. (1995). Stereotypes and ethnocentrism: Diverging interethnic perceptions of African American and White American youth. Journal of Personality and Social Psychology, 69 (3), 460-481.
McConahay, J. B. (1983). Modern racism and modem discrimination. Personality and Social Psychology Bulletin, 9, 551-558.

Monteith, M. J., y Spicer, C. V. (2000). Contents and correlates of Whites' and Blacks' racial attitudes. Journal of Experimental Social Psychology, 36, 125-154.

Navas, M. S., y Rojas, A. J. (2010). Aplicación del Modelo Ampliado de Aculturación Relativa (MAAR) a nuevos colectivos de inmigantes en Andalucía: rumanos y ecuatorianos. Sevilla: Junta de Andalucía.

Navas, M. S., García, M. C., Rojas, A. J., Pumares, P., y Cuadrado, I. (2006). Prejuicio y actitudes de aculturación: la perspectiva de autóctonos e inmigrantes. Psicothema, 18, 187-193.

Navas, M., Rojas, A.J., Pumares, P., Lozano, O.M. y Cuadrado, I. (2010). Perfiles de aculturación según el Modelo de Ampliado de Aculturación Relativa: Autóctonos, inmigrantes rumanos y ecuatorianos. Revista de Psicología Social, 25 (3), 295-312.

Pettigrew, T. F., y Meertens, R. W. (1992). Le racisme voilé: dimensions et mesure. En M. Wieviorka, Racisme et modernité (pp. 109-126). París: La Découverte.

Pettigrew, T. F., y Meertens, R. W. (1995). Subtle and blatant prejudice in Western Europe. European Journal of Social Psychology, 25, 57-75.

Pumares, P., García, A. y Asensio, A. (2006) La movilidad laboral y geográfica de la población extranjera en España. Madrid: Subdirección General de Información Administrativa y Publicaciones, Observatorio Permanente de la inmigración, Ministerio de Trabajo y Asuntos Sociales

Quiles, M. N., y Leyens, J. P. (2003). El prejuicio desde el punto de vista de la víctima. En J. F. Morales, y C. Huici, Estudios de Psicología Social (pp. 119-143). Madrid: UNED.

Rojas, A. J., Navas, M. S., Lozano, O. M., y Gómez-Berrocal, C. (2010). Test de favoritismo endogrupal interétnico adaptado al modelo ampliado de aculturación relativa: fiabilidad y evidencias de validez. Psicothema, 22 (4), 1039-1047.

Rojas, A.J., Navas, M., Lozano, O.M. y Pérez, P.J. (2011). Prejudiced attitude measurement using the Rasch rating scale model. Psychological Reports, 109 (2), 553-572.

Satorra, A., y Bentler, P. M. (1994). Corrections to test statistics and standard errors in covariance structure analysis. En A. V. Eye, y C. C. Clogg, Latent variables analysis: Applications for developmental research (pp. 399-419). Thousand Oaks, CA: Sage.

Schuman, H., Steeh, C., Bobo, L., y Krysan, L. (1997). Racial attitudes in America: Trends and interpretations. Cambridge: Harvard University Press.

Shelton, J. N. (2000). A reconceptualization of how we study issues of racial prejudice. Personality and Social Psychology Review, 4 (4), 374-390.

SPSS Inc. (2006). SPSS 15.0 para Windows. Chicago, IL: SPSS Inc.

Steiger, J. H. (1990). Structural model evaluation and modification: an interval estimation approach. Multivariate Behavioral Research, 25, 173-180.

Swim, J. K., y Stangor, C. (1998). Prejudice: the target's perspective. San Diego, CA: Academic Press.

(Artículo recibido: 27-12-2011, revisado: 6-2-2012, aceptado: 18-2-2012) 